\title{
Efficient Management of 2-d Interval Relations
}

\author{
Nikos Lorentzos and Yannis Manolopoulos \\ Informatics Laboratory, Agricultural University of Athens, 11855 Athens, Greece. \\ Department of Informatics, Aristotle University, 54006 Thessaloniki, Greece.
}

\begin{abstract}
We identify a number of problems concerning the management of interval data and propose efficient algorithms in the case of 2 dimensional interval relations. The approach is of practical importance and has many applications, one of which is spatiotemporal databases.
\end{abstract}

\section{Introduction}

The term interval is quite generic. Time intervals mark the duration of events (the lifespan of a person). Alphabetic intervals have many applications (family names in the range A-C). Given the wide use of intervals, their handling is of major importance. However, there is a number of problems which relate to their management. Such of them were initially identified in research in temporal databases. In particular, the necessity to support temporal data led to the formalisation of many distinct temporal extensions to the relational model [1]. In spite however of the major differences between the various modelling approaches, one characteristic, common to almost all of them, is that the ordinary projection, set-union and set-difference operations are adapted appropriately, in all of them, so as to apply appropriately to data incorporating time intervals. Next, it was identified that the same problems arise in the management of certain types of spatial data [2,3], and this gave recently rise in research in spatiotemporal databases [4].

The Interval-Extended Relational Model (IXRM) was defined to handle them in a uniform way. In this paper we investigate the properties of the IXRM operations and propose efficient algorithms for the above operations. Our work restricts to relations with two pure interval attributes. The algorithms have been based on the geometric interpretation of the contents of pure interval attributes and improve substantially the time and space requirements. The remainder of this work is as follows: In section 2 we identify certain problems concerning the management of interval data. In section 3 we present briefly the IXRM and investigate the properties of its operations. In section 4 we make use of these properties and provide efficient algorithms. Conclusions are drawn in the last section.

\section{Motivation}

In this section we demonstrate the problems concerning the projection, insertion and deletion of interval data. Commercial DBMSs do not support them directly. 
Projection: In relation ASSIGNMENT (figure 1) we record the history of employee assignments to projects. The query "list the time intervals during which each employee was assigned to some project" requires to project out the second attribute of ASSIGNMENT. If the standard projection operation is used to this end, A1 (figure 1) will be obtained. In contrast, the user would rather obtain A2 (figure 1). We say that A2 is a normalised relation, to denote that it does not contain adjacent or overlapping intervals, which data duplication. For example, the fact that John was assigned to some project for each of the dates in [d20,d50), is implicit in A1, from both its first and third tuple.

Assignment
\begin{tabular}{|c|c|c|}
\hline Name & Proj & Time \\
\hline John & P1 & {$[\mathrm{d} 10, \mathrm{~d} 50)$} \\
John & P1 & {$[\mathrm{d} 80, \mathrm{~d} 120)$} \\
John & P2 & {$[\mathrm{d} 20, \mathrm{~d} 80)$} \\
John & P3 & {$[\mathrm{d} 80, \mathrm{~d} 100)$} \\
Alex & P1 & {$[\mathrm{d} 30, \mathrm{~d} 50)$} \\
Alex & P2 & {$[\mathrm{d} 70, \mathrm{~d} 150)$} \\
\hline
\end{tabular}

A1
\begin{tabular}{|c|c|}
\hline Name & Time \\
\hline John & {$[\mathrm{d} 10, \mathrm{~d} 50)$} \\
John & {$[\mathrm{d} 80, \mathrm{~d} 120)$} \\
John & {$[\mathrm{d} 20, \mathrm{~d} 80)$} \\
John & {$[\mathrm{d} 80, \mathrm{~d} 100)$} \\
Alex & {$[\mathrm{d} 30, \mathrm{~d} 50)$} \\
Alex & {$[\mathrm{d} 70, \mathrm{~d} 150)$} \\
\hline
\end{tabular}

A2

\begin{tabular}{|c|c|}
\hline Name & Time \\
\hline John & {$[\mathrm{d} 10, \mathrm{~d} 120)$} \\
Alex & {$[\mathrm{d} 30, \mathrm{~d} 50)$} \\
Alex & {$[\mathrm{d} 70, \mathrm{~d} 150)$} \\
\hline
\end{tabular}

Fig. 1. Relations with interval data.

\begin{tabular}{|c|c|c|c|}
\hline \multicolumn{4}{|c|}{ LAND } \\
\hline Pno & Depth & Time & $\mathrm{pH}$ \\
\hline 1 & {$[0,20)$} & {$[\mathrm{d} 0, \mathrm{~d} 80)$} & \\
\hline 1 & {$[0,100)$} & {$[\mathrm{d} 120, \mathrm{~d} 200)$} & 8.0 \\
\hline 1 & {$[30,120)$} & {$[\mathrm{d} 0, \mathrm{~d} 80)$} & 8.0 \\
\hline 1 & {$[0,30)$} & {$[\mathrm{d} 80, \mathrm{~d} 120)$} & 8.2 \\
\hline 2 & {$[0,60)$} & {$[\mathrm{d} 10, \mathrm{~d} 50)$} & 8.3 \\
\hline 2 & {$[60,100)$} & {$[\mathrm{d} 30, \mathrm{~d} 90)$} & 8.3 \\
\hline
\end{tabular}

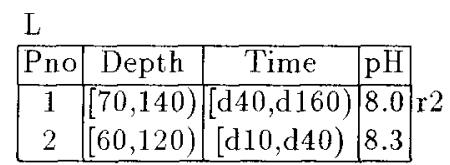

Fig. 2. Relations with two pure interval atributes.

Similar problems also arise in relations with more than one pure interval attribute. For example, LAND (figure 2) has two such attributes, Depth, Time, of an integer, time interval type, respectively. A non-trivial projection of LAND on a set of attributes which include either Depth or Time will yield a nonnormalised relation. Therefore, the projection of a relation with pure interval attributes has to be replaced by some normalisation operation, before the result relation is presented to the user.

Data Insertion: Assume that we want to insert into LAND the contents of $\mathrm{L}$ (figure 2). Using the standard insertion operation, this will result in relation LAND1 (figure 3). LAND1 is non-normalised (for example the soil pH at depth 70 on date $\mathrm{d} 40$, is recorded in both $\mathrm{r} 2$ and $\mathrm{r} 4$ ). In fact, we would like to obtain LAND2 (figure 3), which is normalised.

Data Deletion: If we use the standard deletion operation, to delete from LAND the contents of L, nothing will actually be deleted, whereas we would like to obtain LAND3 (figure 3). 


\section{The Interval Extended Relational Model}

In this section we describe shortly the IXRM, which overcomes the problems identified in the previous section. Its formalisation can be found in [5].

\begin{tabular}{|c|c|c|c|}
\hline Pno & Depth & Time & $\mathrm{pH}$ \\
\hline 1 & {$[0,20)$} & {$[\mathrm{d} 0, \mathrm{~d} 80)$} & 8.0 \\
\hline 1 & {$[70,140)$} & {$[\mathrm{d} 40, \mathrm{~d} 160)$} & 8.0 \\
\hline 1 & {$[0,100)$} & {$[\mathrm{d} 120, \mathrm{~d} 2$} & 8.0 \\
\hline 1 & {$[30,120)$} & {$[\mathrm{d} 0, \mathrm{~d} 80)$} & \\
\hline 1 & {$[0,30)$} & {$[\mathrm{d} 80, \mathrm{~d} 120)$} & 8.2 \\
\hline 2 & {$[0,60)$} & {$[\mathrm{d} 10$} & 8.3 \\
\hline 2 & {$[60,100)$} & {$[\mathrm{d} 30, \mathrm{~d} 90)$} & 8.3 \\
\hline 2 & {$[60,120)$} & {$[\mathrm{d} 10, \mathrm{~d} 40)$} & 8.3 \\
\hline
\end{tabular}

\begin{tabular}{|c|c|c|c|}
\hline \multicolumn{4}{|l|}{$\mathrm{L}$} \\
\hline Pno & Depth & Time & $\mathrm{pH}$ \\
\hline 1 & {$[0,20)$} & {$[\mathrm{d} 0, \mathrm{~d} 80)$} & $8.0 \mathrm{t}$ \\
\hline 1 & {$[30,70)$} & {$[\mathrm{d} 0, \mathrm{~d} 80)$} & $8.0 \mid \mathrm{t}$. \\
\hline 1 & {$[100,120)$} & {$[\mathrm{d} 0, \mathrm{~d} 160)$} & $8.0 \mid \mathrm{t}:$ \\
\hline 1 & {$[70,100)$} & {$[\mathrm{d} 0, \mathrm{~d} 200)$} & $8.0 \mid \mathrm{t}$ \\
\hline 1 & {$[120,140)$} & {$[\mathrm{d} 40, \mathrm{~d} 160)$} & $8.0 \mid \mathrm{t}:$ \\
\hline 1 & {$[0,70)$} & {$[\mathrm{d} 120, \mathrm{~d} 200)$} & $8.0 \mid \mathrm{t} t$ \\
\hline 1 & {$[0,30)$} & {$[\mathrm{d} 80, \mathrm{~d} 120)$} & 8.2 \\
\hline 2 & {$[100,120)$} & {$[\mathrm{d} 10, \mathrm{~d} 40)$} & 8.3 \\
\hline 2 & {$[0,60)$} & {$[\mathrm{d} 10, \mathrm{~d} 50)$} & 8.3 \\
\hline 2 & {$[60,100)$} & {$[\mathrm{d} 10, \mathrm{~d} 90)$} & 8.3 \\
\hline \multicolumn{3}{|c|}{ A3 } & \\
\hline & \multicolumn{2}{|c|}{\begin{tabular}{|l|l|} 
Name Time \\
\end{tabular}} & \\
\hline \multicolumn{2}{|r|}{ John } & d10 & \\
\hline & $\ldots$ & \multirow{2}{*}{$\begin{array}{c}\ldots \\
\mathrm{d} 119\end{array}$} & \\
\hline & John & & \\
\hline & Alex & $\mathrm{d} 30$ & \\
\hline & $\cdots$ & \multirow{2}{*}{$\ddot{d 49}$} & \\
\hline & Alex & & \\
\hline & Alex & $\mathrm{d} 70$ & \\
\hline & Alex & $\begin{array}{c}\cdots \\
\mathrm{d} 149\end{array}$ & \\
\hline
\end{tabular}

Fig. 3. Insertion and deletion of interval data. Result of operation unfold.

A 1-dimensional (1-d) space is a non-empty, finite, totally ordered set D of points: $\mathrm{D}=\mathrm{d}_{1}, \mathrm{~d}_{2}, \ldots, \mathrm{d}_{\mathrm{n}}$. (Without loss of generality, we occasionally start numbering from $d_{0}$.) A (1-d) interval over $D$ is defined as

$$
\left[\mathrm{d}_{\mathrm{m}}, \mathrm{d}_{\mathrm{n}}\right)=\left\{\mathrm{d}_{\mathrm{k}} \mid \mathrm{d}_{\mathrm{m}} \leq \mathrm{d}_{\mathrm{k}}<\mathrm{d}_{\mathrm{n}}, \mathrm{d}_{\mathrm{m}}, \mathrm{d}_{\mathrm{n}} \in \mathrm{D}\right\}
$$

The points $d_{m}, d_{n}$ are the boundaries of $\left[d_{m}, d_{n}\right)$, denoted by start $\left(\left[d_{m}, d_{n}\right)\right)$, stop $\left(\left[\mathrm{d}_{\mathrm{m}}, \mathrm{d}_{\mathrm{n}}\right)\right)$, respectively. An interval $\left[\mathrm{d}_{\mathrm{i}}, \mathrm{d}_{\mathrm{i}+1}\right)$, with exactly one point, is called elementary. The set of all intervals over $D$ is denoted by $I(D)$. Thus, if DATES $=d_{0}, d_{1}, \ldots, d_{200}$ is a set of consecutive dates then $\left[d_{10}, d_{21}\right)$ is an interval in I(DATES). If $\left[\mathrm{d}_{\mathrm{m}}, \mathrm{d}_{\mathrm{n}}\right)$ and $\left[\mathrm{d}_{\mathrm{p}}, \mathrm{d}_{\mathrm{q}}\right)$ are two intervals, we define a predicate, merges, as

$$
\left[\mathrm{d}_{\mathrm{m}}, \mathrm{d}_{\mathrm{n}}\right) \text { merges }\left[\mathrm{d}_{\mathrm{p}}, \mathrm{d}_{\mathrm{q}}\right) \text { if and only if }\left(\mathrm{d}_{\mathrm{n}} \geq \mathrm{d}_{\mathrm{p}} \text { and } \mathrm{d}_{\mathrm{q}} \geq \mathrm{d}_{\mathrm{m}}\right)
$$

If $D_{1}, \ldots, D_{n}$ are spaces then every subset $R$ of the Cartesian product $I\left(D_{1}\right) \times \ldots$ $\mathrm{x} \mathrm{I}\left(\mathrm{D}_{\mathrm{n}}\right)$ is an $n$-d interval relation. Each tuple (element) of $\mathrm{R}$ represents an $n-d$ interval. An n-d interval $\left(\left[\mathrm{d}_{\mathrm{i} 1}, \mathrm{~d}_{\mathrm{i} 1+1}\right),\left[\mathrm{d}_{\mathrm{i} 2}, \mathrm{~d}_{\mathrm{i} 2+1}\right), \ldots,\left[\mathrm{d}_{\mathrm{in}}, \mathrm{d}_{\mathrm{in}+1}\right)\right)$ with exactly one $n$ - $d$ point $\left(\mathrm{d}_{11}, \ldots, \mathrm{d}_{\mathrm{in}}\right)$, is called $n$-d elementary interval. We further notice that a point $d_{i}$ can be seen as an interval $\left[d_{i}, d_{i+1}\right)$. Therefore, if we use the notation $X(D)$ to denote exclusively either $I(D)$ or $D$, then every subset of $X\left(D_{1}\right) \times \ldots x$ 
$\mathrm{X}\left(\mathrm{D}_{\mathrm{n}}\right)$ is, up to an isomorphism, an interval relation. Hence, all the relations in figures 1-3 are interval relations.

Two operations have been formalised in the IXRM: If $R(A, B=I(D))$ is a relation then for each tuple $\left(a,\left[d_{m}, d_{n+1}\right)\right)$ in $R, S=u n f o l d[B](R)$ consists of the set of tuples: $\left(\mathrm{a}, d_{m}\right),\left(\mathrm{a}, d_{m+1}\right),\left(\mathrm{a}, d_{m+2}\right), \ldots,\left(\mathrm{a}, d_{n}\right)$. Conversely, if $\mathrm{S}$ consists of the above tuples, but it does not contain any of $\left(a, d_{m-1}\right)$ and $\left(a, d_{n+1}\right)$, then fold $[\mathrm{B}](\mathrm{S})$ produces from them the single tuple $\left(\mathrm{a},\left[d_{m}, d_{n+1}\right)\right)$. More generally, fold coalesces two or more overlapping or adjacent intervals into one. Examples of these operations, based on the relations in figures 1-3, are

$$
\begin{aligned}
\mathrm{A} 3=u n f o l d[\text { Time }](\mathrm{A} 1), \mathrm{A} 3 & =\text { unfold }[\text { Time }](\mathrm{A} 2), \mathrm{A} 2=\text { fold }[\text { Time }](\mathrm{A} 3), \\
\mathrm{A} 2 & =\operatorname{fold}[\text { Time }](\mathrm{A} 1)
\end{aligned}
$$

If $\left[B_{1}, \ldots, B_{m}\right]$ is any list of the attributes of a relation $R\left(A_{1}, \ldots, A_{n}\right)$, the definitions of unfold and fold are now extended as follows:

$$
\begin{aligned}
& \text { unfold }\left[\mathrm{B}_{1}, \mathrm{~B}_{2}, \ldots, \mathrm{B}_{\mathrm{m}}\right](\mathrm{R})=\text { unfold }\left[\mathrm{B}_{\mathrm{m}}\right]\left(\ldots\left(\text { unfold }\left[\mathrm{B}_{2}\right]\left(\text { unfold }\left[\mathrm{B}_{1}\right](\mathrm{R})\right)\right)\right) \\
& \text { fold }\left[\mathrm{B}_{1}, \mathrm{~B}_{2}, \ldots, \mathrm{B}_{\mathrm{m}}\right](\mathrm{R})=\text { fold }\left[\mathrm{B}_{\mathrm{m}}\right]\left(\ldots\left(\text { fold }\left[\mathrm{B}_{2}\right]\left(\text { fold }\left[\mathrm{B}_{1}\right](\mathrm{R})\right)\right)\right)
\end{aligned}
$$

Another operation, normalise, can also be defined in terms of unfold and fold:

normalise $\left[\mathrm{B}_{1}, \mathrm{~B}_{2}, \ldots, \mathrm{B}_{\mathrm{m}}\right](\mathrm{R})=$ fold $\left[\mathrm{B}_{1}, \mathrm{~B}_{2}, \ldots, \mathrm{B}_{\mathrm{m}}\right]\left(\right.$ unfold $\left.\left[\mathrm{B}_{1}, \mathrm{~B}_{2}, \ldots, \mathrm{B}_{\mathrm{m}}\right](\mathrm{R})\right)$ By the definition of normalise, unfold initially eliminates duplicate tuples. Next fold yields a relation which does not contain adjacent or overlapping intervals.

Finally, if $\mathrm{R}$ and $\mathrm{S}$ be two union-compatible relations, operations unique points set-union (punion) and unique points set-difference (pdiff) are defined as

$$
\begin{aligned}
& \text { punion }\left[\mathrm{B}_{1}, \ldots, \mathrm{B}_{\mathrm{m}}\right](\mathrm{R}, \mathrm{S})=\operatorname{fold}\left[\mathrm{B}_{1}, \ldots, \mathrm{B}_{\mathrm{m}}\right]\left(\text { unfold }\left[\mathrm{B}_{1}, \ldots, \mathrm{B}_{\mathrm{m}}\right](\mathrm{R}) \cup\right. \\
& \text { unfold } \left.\left[\mathrm{B}_{1}, \ldots, \mathrm{B}_{\mathrm{m}}\right](\mathrm{S})\right) \\
& \text { pdiff }\left[\mathrm{B}_{1}, \ldots, \mathrm{B}_{\mathrm{m}}\right](\mathrm{R}, \mathrm{S})=\text { fold }\left[\mathrm{B}_{1}, \ldots, \mathrm{B}_{\mathrm{m}}\right]\left(\text { unfold }\left[\mathrm{B}_{1}, \ldots, \mathrm{B}_{\mathrm{m}}\right](\mathrm{R})\right. \text { - } \\
& \text { unfold } \left.\left[\mathrm{B}_{1}, \ldots, \mathrm{B}_{\mathrm{m}}\right](\mathrm{S})\right)
\end{aligned}
$$

By their definition, these operations return a normalised relation. It is easy to show that

$$
\text { punion }\left[\mathrm{B}_{1}, . ., \mathrm{B}_{\mathrm{m}}\right](\mathrm{R}, \mathrm{S})=\text { fold }\left[\mathrm{B}_{1}, . ., \mathrm{B}_{\mathrm{m}}\right]\left(\text { unfold }\left[\mathrm{B}_{1}, . ., \mathrm{B}_{\mathrm{m}}\right](\mathrm{R} \cup \mathrm{S})\right) \text {. }
$$

Using the above operations, the problems identified in the previous section are faced as follows:

Normalisation: $\mathrm{A} 2=$ normalise $[$ Time $](\mathrm{A} 1)$,

LAND2 = normalise $[$ Time,Depth] $(\mathrm{LAND} 1)$

Insertion of Interval Data: LAND2 = punion[Time,Depth](LAND, L)

Deletion of Interval Data: LAND3 $=p \operatorname{diff[Time,Depth](LAND,L)}$

The above three operations can be used to handle relations with arbitrarily many pure interval attributes. However, their definition in terms of unfold, makes them prohibitively costly. In the sequel we investigate the properties of unfold and fold and provide efficient algorithms in the next section.

An interval $\left[d_{m}, d_{n}\right)$ can geometrically be seen as a line segment which is closed to the left and open to the right. Hence, every tuple of an $n$-ary relation can be seen as an $n-d$ cuboid. Thus, the single tuple of relation $R$ (figure $4(a)$ ) is represented by the orthogonal rectangle WXYZ (figure 4(b)). The 2-d points on sides $X Y$ and $Y Z$ are not points of the interval. In contrast, the 2 -d points in the 
rectangle and also those on sides $\mathrm{WX}$ and $\mathrm{WZ}$ (excluding $\mathrm{X}$ and $\mathrm{Z}$ ) are points of it. If $\mathrm{R} 1=$ unfold $[\mathrm{B}](\mathrm{R})$ is now issued, then $\mathrm{R} 1$ will consist of four tuples, $([1,4)$, $i), i=1,2,3,4$. Since point $i$ is isomorphic with the interval $[i, i+1)$, each of these tuples can geometrically be interpreted by one of the four adjacent rectangles in figure 4(c). This figure shows that unfold $[\mathrm{B}](\mathrm{R})$ splits each tuple (interval) of a relation $R$ into intervals whose values for attribute $B$ are elementary intervals. Similarly, if we issue next $\mathrm{R} 2=$ unfold $[\mathrm{A}](\mathrm{R} 1)$ we obtain a relation consisting of 2 -d elementary points whose geometric interpretation is shown in figure $4(d)$.

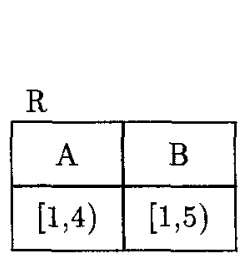

(a)

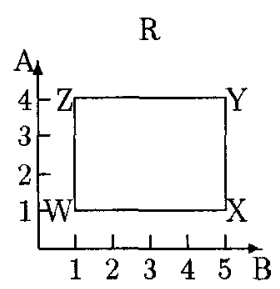

(b)

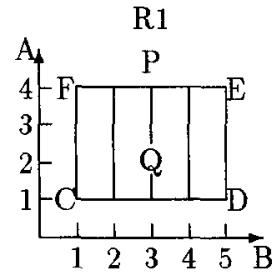

(c)

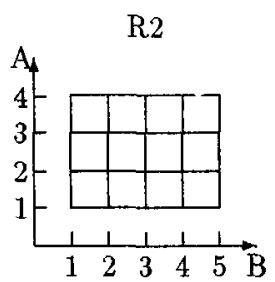

(d)

Fig. 4. Geometric interpretation of a 2 -d interval and of operations unfold and fold.

It can easily be seen that the relations, whose representation is given in figure 4 , satisfy $\mathrm{R} 1=$ fold $[\mathrm{A}](\mathrm{R} 2), \mathrm{R}=$ fold $[\mathrm{B}](\mathrm{R} 1)$ and, therefore, $\mathrm{R}=$ fold $[\mathrm{A}, \mathrm{B}](\mathrm{R} 2)$. The following properties can be deduced from the definition of fold (the reader can found their formal proofs in [6]).

Property 1: unfold $[\mathrm{B}, \mathrm{A}](\mathrm{R})=$ unfold $[\mathrm{A}, \mathrm{B}](\mathrm{R})$.

Property 2: If a relation $R(A, B)$ is sorted with respect to $A$ and $B$ then fold $[\mathrm{B}](\mathrm{R})$ can be accomplished in one pass, by scanning sequentially the tuples of $R$.

Property 3: If $R_{1}, R_{2}, \ldots, R_{k}$ is a partition of $R(A, B)$ such that all the tuples in the same $R_{i}$ have identical $A$ values whereas tuples in distinct $R_{i}$ and $R_{j}$ have different $A$ values, then

$$
\text { fold }[\mathrm{B}](\mathrm{R})=\text { fold }[\mathrm{B}]\left(\mathrm{R}_{1}\right) \cup \text { fold }[\mathrm{B}]\left(\mathrm{R}_{2}\right) \cup \ldots \cup \text { fold }[\mathrm{B}]\left(\mathrm{R}_{\mathrm{k}}\right) \text {. }
$$

Property 4: Let $R(A, B=I(D))$ be a relation and $B P$ a subset of points in $D$. If $S$ is the relation obtained from $R$ if all the tuples of $R$ are split with respect to the points in $\mathrm{BP}$, then

$$
\text { fold }[\mathrm{B}](\mathrm{R})=\text { fold }[\mathrm{B}](\mathrm{S}) \text {. }
$$

Property 5: fold $[\mathrm{B}](\mathrm{R})=$ fold $[\mathrm{B}]($ unfold $[\mathrm{B}](\mathrm{R}))$.

Property 6: Let $R(A, B=I(D))$ be a relation and $B P$ be a subset of points in $D$. If $S$ is the relation obtained from $R$ if all the tuples of $R$ are split with respect to the points in $\mathrm{BP}$, then

$$
\text { fold }[\mathrm{B}](\text { unfold }[\mathrm{B}](\mathrm{R}))=\text { fold }[\mathrm{B}](\mathrm{S}) \text {. }
$$

Property 7: For any relation $R$,

$$
\text { fold }[\mathrm{A}, \mathrm{B}](\text { unfold }[\mathrm{A}, \mathrm{B}](\mathrm{R}))=\text { fold }[\mathrm{A}, \mathrm{B}](\text { unfold }[\mathrm{B}](\mathrm{R})) \text {. }
$$

Because of the above properties normalise and punion are equivalent to normalise $\left[\mathrm{B}_{1}, \mathrm{~B}_{2}, \ldots, \mathrm{B}_{\mathrm{m}}\right](\mathrm{R})=$ fold $\left[\mathrm{B}_{1}, \mathrm{~B}_{2}, \ldots, \mathrm{B}_{\mathrm{m}}\right]\left(\right.$ unfold $\left.\left[\mathrm{B}_{2}, \mathrm{~B}_{3}, \ldots, \mathrm{B}_{\mathrm{m}}\right](\mathrm{R})\right)$ punion $\left[\mathrm{B}_{1}, \ldots, \mathrm{B}_{\mathrm{m}}\right](\mathrm{R}, \mathrm{S})=$ fold $\left[\mathrm{B}_{1}, \ldots, \mathrm{B}_{\mathrm{m}}\right]\left(\right.$ unfold $\left.\left[\mathrm{B}_{2}, \mathrm{~B}_{3}, \ldots, \mathrm{B}_{\mathrm{m}}\right](\mathrm{R} \cup \mathrm{S})\right)$. 


\section{Efficient Algorithms}

Using the properties of the previous section, we now provide efficient algorithms for normalise, punion and pdiff.

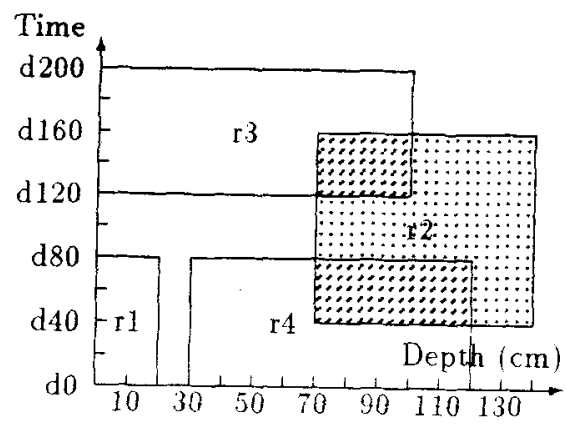

(a)

$\mathrm{bd} 2$

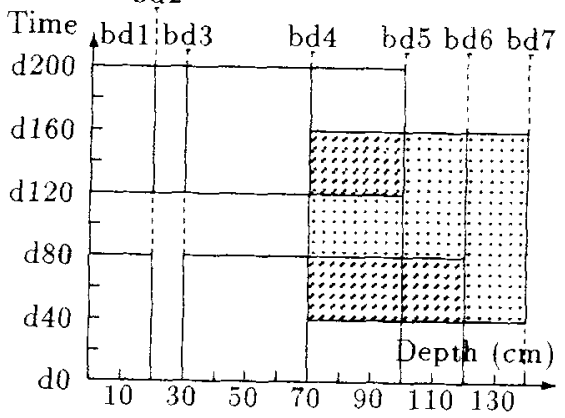

(c)

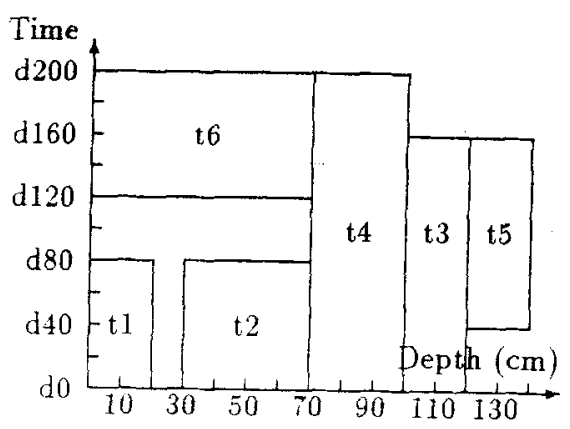

(b)

\section{$b \mathrm{~d} 2$}

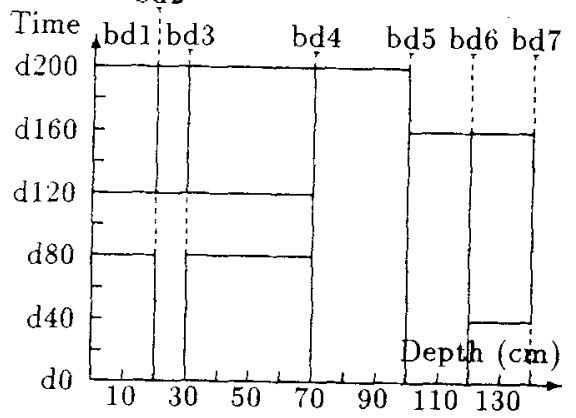

(d)

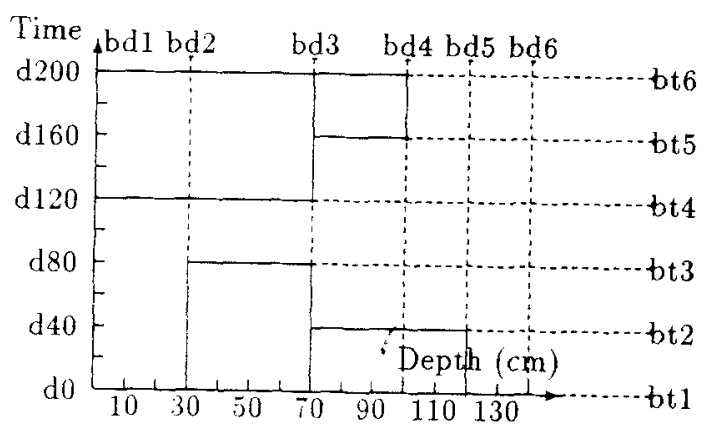

(e)

Fig. 5. Geometric interpretation of efficient operations. 


\subsection{Efficient Normalization of 2-d Interval data}

LAND1 (figure 3) is non-normalised, therefore normalise/fold has to be applied. From property 3 it suffices to normalise each distinct set in the partition of LAND1. Indeed, LAND1 is already sorted with respect to Pno and $\mathrm{pH}$ and the partition is $\{P, Q, R\}$, where $P$ consists of the first four tuples, $Q$ consists of the fifth and $\mathrm{R}$ contains consists of the last three tuples. The interpretation of $\mathrm{P}$ is shown in figure 5(a). Tuple $\mathrm{r} 2$, in particular, is dark. The rectangles in $\mathrm{r} 2$ which are darker, including their bottom and left sides, denote duplicate points.

If we now issue normalise[Time,Depth] $(\mathrm{P})$, we shall obtain the first six tuples of LAND2 (figure 3). The interpretation of these tuples is shown in figure 5 (b). By using property 7, the number of attributes on which LAND1 has to be unfolded may by reducing by one, that is normalise[Time,Depth](LAND1) = fold[Time,Depth](unfold[Depth](LAND1)). If we issue unfold[Depth](LAND1), each of the rectangles in figure 5(a) will be split into a large number of rectangles with Depth components of exactly one point each. The same result can be obtained if the rectangles in figure 5(a) are split only into sub-rectangles with respect to the boundary points of their Depth values. This split is shown in figure $5(\mathrm{c})$. The set of these boundary points with respect to which the split will take place, is DepthBoundaries $=\{\mathrm{bd} 1, \ldots, \mathrm{bd} 7\}$. Hence, rather than using unfold, we can use this split, to finally achieve an equivalent result. Thus, if $\mathrm{P} 1$ is the relation having as tuples the rectangles in figure $5(\mathrm{c})$, then the interpretation of $\mathrm{P} 2=f o l d[$ Time $](\mathrm{P} 1)$ is shown in figure $5(\mathrm{~d})$. Finally, $\mathrm{P} 3=$ fold $[\mathrm{Depth}](\mathrm{P} 2)$ shown in figure $5(\mathrm{~b})$, is the interpretation of the normalised relation LAND2. The above procedure has to be repeated for each set in the partition $\{P, Q, R\}$ of LAND1.

Input : Relation $\mathrm{R}(\mathrm{NI}, \mathrm{A}, \mathrm{B})$, Index(NI) of relation $\mathrm{R}$.

Output: Relation $\mathrm{S}=$ normalise $[\mathrm{A}, \mathrm{B}](\mathrm{R})$.

begin

for each distinct ni value stored in Index(NI) do

begin

retrieve the subset $\mathrm{Ri}$ of $\mathrm{R}$ with $\mathrm{Ri}[\mathrm{NI}]=\mathrm{ni}$

for each tuple $\mathbf{r}(\mathbf{n i}, \mathrm{a}, \mathrm{b})$ of Ri do

begin

store the boundaries of $\mathrm{b}$ in BBoundaries(B)

end

store $(\mathrm{a}, \mathrm{b})$ in $\operatorname{Temp}(\mathrm{A}, \mathrm{B})$

sort $\mathrm{BBoundaries}(\mathrm{B})$ and eliminate any duplicate rows

split each tuple in $\operatorname{Temp}(\mathrm{A}, \mathrm{B})$ wrt the points in BBoundaries(B)

fold(Temp)

for each tuple $r(a, b)$ in Temp(A,B), write $S(n i, a, b)$ end

end Table 1: Algorithm for Efficient-normalise on two attributes.

Let $\mathrm{NI}$ be a set of zero or more attribute and let $\mathrm{R}(\mathrm{NI}, \mathrm{A}=\mathrm{I}(\mathrm{D} 1), \mathrm{B}=\mathrm{I}(\mathrm{D} 2))$ be a relation. We assume that two temporary files, $B B$ oundaries $(B=D 2)$ and 
$\operatorname{Temp}(A=I(D 1), B=I(D 2))$ can fit in main memory. For simplicity, we assume also that the result of a split or fold in a main memory structure, is stored in this same structure. The algorithm normalise, shown in table 1, calls algorithm fold, given in table 2 .

Input : Relation $\operatorname{Temp}(\mathrm{A}, \mathrm{B})$.

Output: Relation fold $[\mathrm{A}, \mathrm{B}]($ Temp).

begin

sort $\operatorname{Temp}(\mathrm{A}, \mathrm{B})$ on $\mathrm{B}, \mathrm{A}$

fold $\operatorname{Temp}(\mathrm{A}, \mathrm{B})$ on $\mathrm{A}$

sort $\operatorname{Temp}(\mathrm{A}, \mathrm{B})$ on $\mathrm{A}, \mathrm{B}$

end

fold $\operatorname{Temp}(\mathrm{A}, \mathrm{B})$ on $\mathrm{B}$

Table 2: Algorithm to fold on two attributes.

\subsection{Efficient Insertion of 2-d Interval Data}

Consider LAND (figure 2), indexed on $\mathrm{NI}=[$ Pno, $\mathrm{pH}]$ and assume that we want to insert into L (figure 2). By using property 3, we initially partition $\mathrm{L}$ with respect to its distinct $\mathrm{NI}$ values and for each set in this partition we consider only the respective partition in LAND. More precisely, $L$ can be partitioned into two sets, of one tuple each. The Pno and $\mathrm{pH}$ values of the tuples in the first set (tuple r2) are 1 and 8.0, respectively. For this set, it suffices to consider from LAND only those tuples with the same respective values. However, tuple r1 (figure 5(a)) can in fact be excluded because neither its Time nor its Depth component merges with the respective component of $\mathrm{r} 2$. In contrast, tuples $\mathrm{r} 3$ and $\mathrm{r} 4$ of LAND have to be involved in punion because: (i) Their Pno and $\mathrm{pH}$ values are identical with the respective values of $\mathbf{r} 2$ (also satisfied by $\mathrm{r} 1$ ) and (ii) both their Depth and Time values merge with the respective values of $\mathrm{r} 2$ (not satisfied by $\mathrm{r} 1$ ).

Input : Relations $\mathrm{R}(\mathrm{NI}, \mathrm{A}, \mathrm{B})$, Index(NI) of relation $\mathrm{R}, \mathrm{T}(\mathrm{NI}, \mathrm{A}, \mathrm{B})$

( ${ }^{*} \mathrm{R}$ is normalised with respect to $A, B{ }^{*}$ )

Output: Relation $\mathrm{R}=$ punion $[\mathrm{A}, \mathrm{B}](\mathrm{R}, \mathrm{T})$

begin

sort $\mathrm{T}$ on $\mathrm{NI}$

for each subset $\mathrm{Ti}(\mathrm{NI}, \mathrm{A}, \mathrm{B})$ of $\mathrm{T}$ with identical NI values do begin

for each tuple r(ni,a2,b2) of Ti do

begin

store the boundaries of $\mathrm{b} 2$ in BBoundaries(B)

store $(\mathrm{a} 2, \mathrm{~b} 2)$ in $\operatorname{Temp}(\mathrm{A}, \mathrm{B})$

for each tuple $r(n i, a 1, b 1)$ in $R$ such that

(a1 merges a2) and (b1 merges b2) do

begin

store the boundaries of $\mathrm{b} 1$ in BBoundaries(B)

store $(\mathrm{a} 1, \mathrm{~b} 1)$ in $\operatorname{Temp}(\mathrm{A}, \mathrm{B})$ 


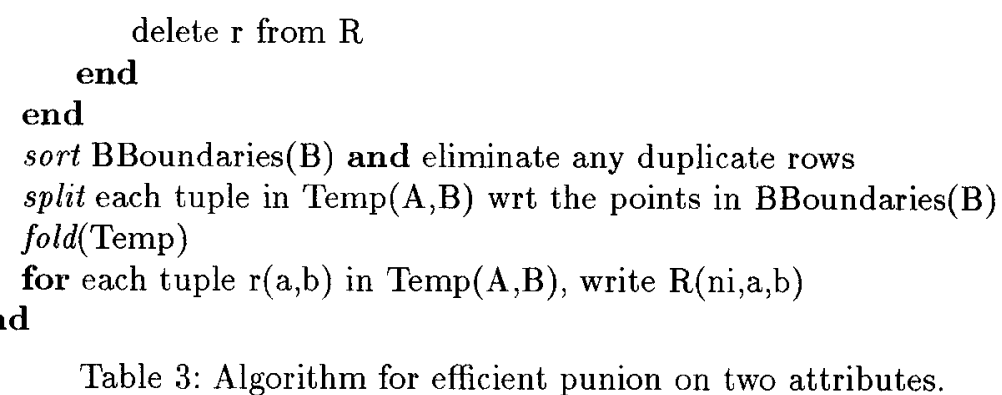

It is now clear from sub-section 4.1, that if punion [Time,Depth] (LAND,L) is issued, the resulting relation will contain the tuples $t 2-t 6$ (figure $5(\mathrm{~b})$ ), in place of tuples $\mathrm{r} 3$ and $\mathrm{r} 4$ (figure $5(\mathrm{a})$ ). This means that tuples $\mathrm{r} 3$ and $\mathrm{r} 4$ have to be deleted from LAND and tuples t2-t6 will next have to be inserted into LAND. Furthermore, since only the tuples r2-r4 need be split, the set of the Depth boundary points with respect to which the split will take place, is DepthBoundaries = $\{\mathrm{bd} 1, \mathrm{bd} 3, \ldots, \mathrm{bd} 7\}$ (figure $5(\mathrm{c})$ ).

Finally, the tuples derived by this split have to be folded on Time and Depth and be inserted into LAND. The same procedure has to be repeated with each set into which $\mathrm{L}$ is partitioned. The above imply the algorithm for punion which is given in table 3 .

\subsection{Efficient Deletion of 2-d Interval Data}

Now assume that we want to delete from LAND the contents of L (figure 2). Since pdiff involves fold, similarly to punion, L has to be partitioned with respect to the values of its tuples on attributes NI. Then pdiff[Time,Depth](LAND,L) can be implemented by considering separately each distinct set in this partition, with the respective partition in LAND. The first set in the partition of L consists of tuple r2, only. The respective set of LAND consists again of the tuples $r 1, r 3$, $\mathrm{r} 4$ from which only $\mathrm{r} 3$ and $\mathrm{r} 4$ have again to be considered because (i) their Pno and $\mathrm{pH}$ values match with the respective values of $\mathrm{r} 2$ and (ii) both their Depth and Time values merge with the respective values of $r 2$. As can be seen in figure $5(\mathrm{a})$, only the portion in dark of tuples $\mathrm{r} 3$ and $\mathrm{r} 4$ has to be eliminated. Figure $5(\mathrm{e})$ shows the tuples which LAND will contain in place of $\mathrm{r} 3$ and $\mathbf{r} 4$, after the execution of $p$ diff. This implies that (i) once $r 3$ and $r 4$ have been identified, they have to be deleted from LAND and (ii) once the tuples in figure 5(e) have been computed, they have to be inserted into LAND. The tuples in figure 5(e) can be computed as follows:

Input : Relations $\mathrm{R}(\mathrm{NI}, \mathrm{A}, \mathrm{B})$, Index(NI) of relation $\mathrm{R}, \mathrm{T}(\mathrm{NI}, \mathrm{A}, \mathrm{B})$

( $\mathrm{R}$ is normalised with respect to $\mathrm{A}, \mathrm{B}^{*}$ ).

Output: Relation $\mathrm{R}=p \operatorname{diff}[\mathrm{A}, \mathrm{B}](\mathrm{R}, \mathrm{T})$.

begin

sort $\mathrm{T}$ on $\mathrm{NI}$

for each subset $\mathrm{Ti}(\mathrm{NI}, \mathrm{A}, \mathrm{B})$ of $\mathrm{T}$ with identical NI values do 


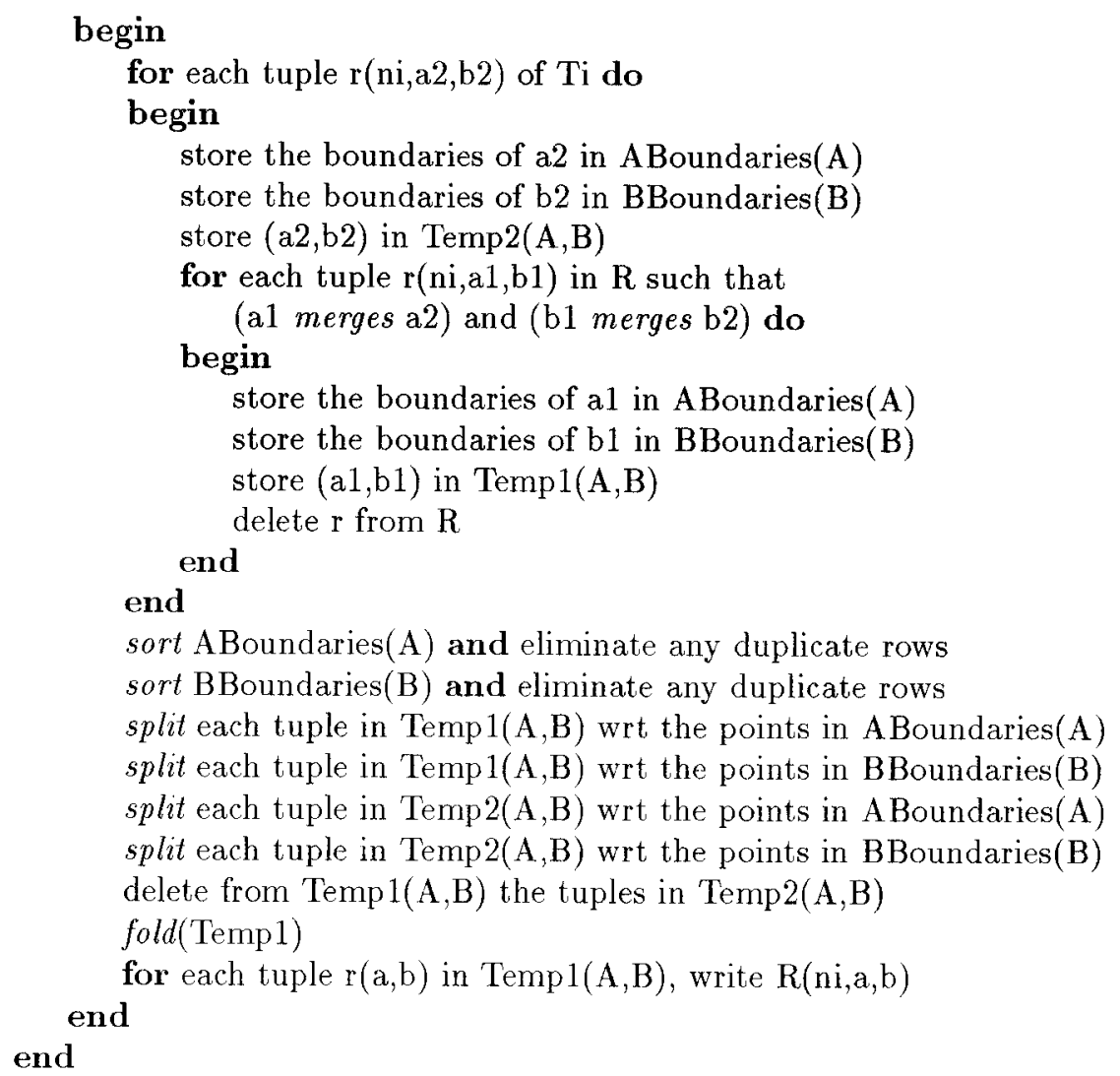

Table 4: Algorithm for efficient pdiff on two attributes.

Firstly, by comparing figures $5(\mathrm{a})$ and $5(\mathrm{e})$, it is clear that, initially, tuples r2-r4 have to be split twice: The first split will be with respect to their Depth boundary points, DepthBoundaries $=\{\mathrm{bd} 1, \ldots, \mathrm{bd} 6\}$. The tuples obtained this way, will next have to be split with respect to their Time boundary points, TimeBoundaries $=\{\mathrm{bt} 1, \ldots, \mathrm{bt} 6\}$. Thus, two distinct main storage structures have now to be maintained, DepthBoundaries and TimeBoundaries. The result obtained after the split of $\mathrm{r} 3$ and $\mathrm{r} 4$ is maintained in a main storage structure, Temp1(A,B). Similarly, the result obtained by the split of $r 2$ is maintained in a main storage structure Temp2(A,B).

Secondly, in order that the dark rectangles of figure $5(\mathrm{c})$ are eliminated, we have to issue

$$
\operatorname{Temp1}(\mathrm{A}, \mathrm{B})=\operatorname{Temp} 1(\mathrm{~A}, \mathrm{~B})-\operatorname{Temp} 2(\mathrm{~A}, \mathrm{~B}) \text {. }
$$

Finally, the result of fold[Time,Depth](Temp1), shown in figure 5(e), has to be inserted into LAND. Hence, the algorithm for an efficient pdiff $[\mathrm{A}, \mathrm{B}]$ is the one given in table 4 . 


\section{Conclusions}

We considered relations with two interval attributes and examined the problems arising when certain operations are applied to them. At the logical level, it was shown how the relational algebra should be extended to overcome these problems, by using two additional operations, fold and unfold. We investigated the properties of these operations and presented efficient algorithms for the management of 2-d interval data. Work concerning their implementation can be found in [6]. In our approach we considered indices on non-interval attributes but spatiotemporal indexing approaches have also been proposed (see for example $[7,8,9]$ for surveys). However, the indexing of interval data is still an open research problem. Thus, for the problems examined in this work, even more efficient algorithms have to be investigated.

\section{Acknowledgment}

This work has been funded by the ESPRIT III Project ORES, 7224.

\section{References}

1. L.E. McKenzie and R.T. Snodgrass: Evaluation of Relational Algebras Incorporating the Time Dimension in Databases, ACM Computing Surveys 23(4), 501-543, 1991.

2. C.S. Jensen and R.T. Snodgrass: Extending Normal Forms to Temporal Relations, TR 92-17, Computer Science Dept., University of Arizona, 1992.

3. S.K. Gadia: Parametric Databases: Seamless Integration of Spatial, Temporal, Belief and Ordinary Data, ACM SIGMOD RECORD 22(1) 15-20, 1993.

4. K.K. Al-Taha, R.T. Snodgrass and M.D. Soo: Bibliography on Spatiotemporal Databases, ACM SIGMOD Record 22(1), 59-67, 1993.

5. N.A. Lorentzos: The Interval Extended Relational Model and its Application to Valid Time Databasesr, in Theory, Design and Implementation (ed. A. Tansel et.al.), Benjamin/Cummings, 67-91, 1993.

6. N.A. Lorentzos, A. Poulovassilis and C. Small: Implementation of Update Operations for Interval Relations, The Computer Journal, 37(3), 164-176, 1994.

7. Y. Manolopoulos and G. Kapetanakis: Overlapping B+trees for Temporal Data, Proc. 5th JCIT Conference, 491-498, 1990.

8. H. Samet: The Design and Analysis of Spatial Data Structures, Addison-Wesley, Reading MA, 1990.

9. C. Kolovson: Indexing for Historical Databases, in Theory, Design and Implementation (ed. A. Tansel et.al.), Benjamin/Cummings, 418-432, 1993. 